\title{
Meaning conveyed by vowels: Some reanalyses of word norm data
}

\author{
ALBERT N. KATZ \\ University of Western Ontario, London, Ontario, Canada
}

\begin{abstract}
R. Tarte and his associates have obtained evidence for phonetic symbolism-like effects in which the critical meaning-bearing unit appears to be the vowel sound, with frequency of vocal resonance being especially implicated. In the present study, word norm data were analyzed with respect to hypotheses generated by Tarte's work. Different monosyllabic words with single embedded vowels were examined with respect to normative data on potency, activity, evaluativeness, roundness, and size. "Phonetic" symbolism-like effects were observed for the potency and activity dimensions and, marginally, for roundness. It appears that, at least for some dimensions, meaning is in part conveyed by superficial nonsemantic aspects of the visually presented word. However, it is unlikely that the present results are due to vowel resonance frequency, because only the potency dimension differences were consistent with that hypothesis.
\end{abstract}

It is generally well accepted that words can be independently processed along a set of discrete dimensions, such as those based on the word's sound or its meaning (e.g., Craik \& Tulving, 1975). The general assumption appears to be that the meaning of a word and its phonetic representation are only accidentally related. In contrast to this position is the phonetic symbolism hypothesis. According to this theory, the phonetic representation of a word actually does convey some meaning. Indeed an extreme version of this position holds that sounds have symbolic connotations that are the same in all languages. Although this extreme position has little empirical support, the more modest position, that within a given language one can find reliable sound-meaning relationships, is still viable (see reviews by Brown, 1958; Taylor, 1976; Taylor \& Taylor, 1965). Confirmation of the existence of this phenomenon should have theoretical implication for a range of problems: the nature of access to semantic memory, the basis for aesthetic reactions to linguistic constructions (such as in the comprehension and appreciation of metaphor), discerning the components of a word which are processed automatically, and, in fact, all cases in which a word's meaning might be influenced by its sound.

In recent years, the existence of phonetic symbolism in the English language has been put on a stronger empirical basis by Tarte and his colleagues. Tarte's general procedure has been to present sound stimuli (either tones or spoken monosyllables) and have people either rate these sounds on semantic-differential scales or match them to geometric forms. Two consistent findings emerge. The

Preparation of this paper was supported by Natural Sciences and Engineering Research Council of Canada Grant No. A7040. I would like to thank Michael O'Boyle for stimulating my interest in phonetic symbolism and Allan Paivio for kindly making his size norms available to me. The author's mailing address is: Department of Psychology, University of Western Ontario, London, Ont., Canada N6A 5C2. first is evidence supporting the presence of phonetic symbolism: sounds are rated or matched to stimuli in a consistent manner. Second, in confirmation of Brown (1958), the sound unit of importance for conveying meaning appears to be the vowel sound (see, e.g., Tarte \& Barritt, 1971). Subsequent work has isolated frequency as a critical aspect of the vowel-related effect (O'Boyle \& Tarte, 1980; Tarte, 1982; Tarte \& O'Boyle, 1982). Highcharacteristic frequency (for instance the vowel /i/) appears to connote high activity and low potency, and to denote smallness and angularity. Low-characteristic frequencies $(/ \mathrm{a} /, / \mathrm{u} /)$ appear to connote greater potency and less activity, and to denote large and round objects.

In the present paper, the presence of phonetic symbolism-like effects will be considered by reanalyzing a set of relevant norms. In all cases, the norms were gathered on words that the participants read and then rated. The logic of the analyses reported here is simple. In all cases, word norms relevant to the types of dimensions studied by Tarte and his colleagues were identified. Then, in order to emphasize vowel effects, all the monosyllabic words containing single embedded vowels were isolated in each normative source. The basic analysis was a single-factor 5(vowel)-level ANOVA. Since the words are not chosen on the basis of any semantic or meaning-based feature or set of features, there is no a priori reason to expect systematic variation between these groups. Finding such differences would indicate that systematic difference in meaning was based on phonetic (or other nonsemantic) features correlated with vowel properties.

\section{SEMANTIC DIFFERENTIAL DATA}

The Heise (1965) norms for 1,000 high-frequency words were consulted for evaluation of the hypothesis that phonetic symbolism conveys connotative meaning. In the 
most extensive test of this hypothesis, Tarte (1982) found that the embedded vowel sound $/ \mathrm{i} /$ was rated as active but not potent, /a/ sounds were rated potent, and / $\mathrm{u} /$ sounds passive. Evaluative scales were inconsistent, but the tendency was for $/ \mathrm{i} /$ to be rated as "better" than $/ \mathrm{u} /$ and $/ \mathrm{a} /$. These differences were tied to the frequency of vocal resonance. Tarte's data were based on the ratings of nine monosyllables, all but one of which were nonwords.

Heise's (1965) norms were selected because they contain the largest number of words scaled on the three dimensions identified by Osgood (1962) and because each dimension was highly reliable, based on factor scores derived from multiple scales for each dimension. Two hundred seventy-six words met the selection criteria for analysis. The breakdown was 86 monosyllables containing the vowel A, 39 Es, 53 Is, 69 Os, and 29 Us. The mean factor score and standard deviation for each vowel group on the connotative dimensions of activity, evaluativeness, and potency are depicted in the upper panel of Table 1. The factor scores are standardized such that positive scores mean active, good, and strong for the three dimensions respectively. A 5 (vowel group) $\times 3$ (repeated-factor semantic-differential-factor scale) ANOVA revealed a significant effect only for the interaction $[F(8,542)=2.29, p<.05]$. Post hoc multiplerange analyses indicated that the vowel groups did not differ with respect to the evaluative dimensions. However, on the activity scale, "I" words were rated most active and words containing " $E$ " least active. This difference was significant $(p<.05)$. On the potency scale, words containing the vowel " $A$ " were rated most potent and those containing the letter " $I$ " least potent; this contrast also was significant $(p<.05)$.

In summary, then, words sampled by vowel types (and not according to meanings) systematically differed on the connotative meanings they aroused. The differences were generally in accord with those observed by Tarte; that is, the greatest differences were observed with vowels differ- ing in vocal resonance frequency. This is most obvious for the potency dimension but is also suggested by the activity dimension data: examination of only the three vowels studied by Tarte results in the same ordering, albeit nonsignificant, on the activity scale that he observed: " $I$ " being most active, " $A$ " intermediate, and " $U$ " least active.

\section{ANGULARITY DATA}

Tarte and Barritt (1971) had participants match CVC trigrams to different figures. They observed that the sound /I/ was matched with the most angular objects (triangles) and $/ \mathrm{U} /$ with the roundest ones (ellipses). Once again, note the correspondence between vowel type and frequency differences. O'Boyle and Tarte (1980) confirmed this correspondence in a study involving the matching of geometric forms to pure tone frequencies. Round figures were consistently assigned lower frequencies.

In the present analyses, unpublished roundness norms, gathered by myself and Marc Marschark of the University of North Carolina-Greensboro, were consulted. These norms were gathered for an envisioned set of experiments on lexical marking and consisted of 325 concrete words rated on a 7 -point roundness scale $(1=$ round) by 31 participants. Sixty-four words met the selection criteria for analysis: 18 words with A, 8 Es, 6 Is, 17 Os, and 15 Us. The mean (scaled such that $1=$ round) and standard deviation for each vowel group are presented in the middle panel of Table 1. A one-way ANOVA indicated a marginal effect of vowel type $[F(4,59)=2.224, p=.077]$. Post hoc multiple-range statistics indicated that only the two extreme groups (U and E) differed from one another $(p<.05)$. At best, then, there is a suggestion in the data that monosyllabic words tend to differ as a function of their embedded vowels. While the low-frequency " $U$ " words were seen as being the roundest (consistent with Tarte \& Barritt, 1971), these words did not differ from

Table 1

Mean Potency, Activity, Evaluativeness, Roundness, and Size Appraisal as a Function of Embedded Vowel

\begin{tabular}{|c|c|c|c|c|c|c|c|c|c|}
\hline \multicolumn{10}{|c|}{ Vowel } \\
\hline \multicolumn{2}{|c|}{ A } & \multicolumn{2}{|c|}{$\mathrm{E}$} & \multicolumn{2}{|c|}{ I } & \multicolumn{2}{|c|}{$\mathrm{O}$} & \multicolumn{2}{|c|}{$\mathrm{U}$} \\
\hline Mean & SD & Mean & SD & Mean & SD & Mean & SD & Mean & SD \\
\hline \multicolumn{10}{|c|}{ Potency Dimension } \\
\hline 0.194 & 1.10 & -0.204 & 0.478 & -0.367 & 1.197 & 0.035 & 0.996 & 0.122 & 0.802 \\
\hline \multicolumn{10}{|c|}{ Activity Dimension } \\
\hline 0.181 & 0.973 & -0.171 & 0.806 & 0.267 & 1.074 & -0.093 & 1.093 & 0.017 & 0.812 \\
\hline \multicolumn{10}{|c|}{ Evaluativeness Dimension } \\
\hline-0.102 & 1.082 & 0.059 & 0.939 & 0.060 & 1.140 & -0.179 & 0.980 & -0.218 & 1.113 \\
\hline \multicolumn{10}{|c|}{ Roundness Dimension } \\
\hline 5.056 & 0.914 & 5.876 & 1.010 & 4.472 & 1.730 & 5.020 & 1.353 & 4.258 & 1.644 \\
\hline \multicolumn{10}{|c|}{ Size Dimension } \\
\hline 3.696 & 1.880 & 3.404 & 1.482 & 3.630 & 2.573 & 3.260 & 1.331 & 3.746 & 2.451 \\
\hline
\end{tabular}


high-frequency "I" words but did differ from words with the embedded vowel "E."

\section{SIZE DATA}

Several studies have indicated that small figures tend to be associated with higher frequency tones or vowels than are large figures (O'Boyle \& Tarte, 1980; Tarte \& Barritt, 1971; Tarte \& O'Boyle, 1982). In the present investigation of this possibility with word monosyllables, unpublished norms gathered by Allan Paivio of the University of Western Ontario were sampled. These norms were based on a sample size of 49 and consisted of 304 concrete words rated on a 9-point scale $(1=$ small). The words have been employed in a series of sizecomparison experiments (see Paivio, 1975). Sixty items (18 As, 7 Es, 7 Is, 14 Os, and 14 Us) met the selection criterion for analysis; the means and standard deviations are presented in the lower panel of Table 1. One-way ANOVA of this data indicated absolutely no support for a "phonetic symbolism"' effect $[F(4,55)=0.146, p=$ .96].

\section{CONCLUSION}

Moderate "phonetic" symbolism effects were observed only with the connotative meaning dimensions of potency and activity. When denotative meaning dimensions (size and angularity) were examined, there was little evidence for the effect. There was a marginal effect for the assignment of angularity but none for that of size. Even when phonetic effects were observed, the data was only partially consistent with a frequency interpretation. Frequency-like effects were found when potency attributions were assessed. No other consistent frequency-like effects emerged. Words with the (high-frequency) embedded vowel "I" were seen as being most active, and those with the (low-frequency) vowel " $\mathrm{A}$ " were associated with roundness, as predicted. On the other hand, the major contrast in both of these cases was not with words having vowels on the other end of the frequency dimension but rather with embedded " $E$ " words.

Taken together, these analyses suggest that at least certain general dimensions of meaning in single-syllable words (potency, activity, and roundness) are possibly conveyed, in part, by the nature of the embedded vowel. The link between the weak effects shown here and frequency differences is extremely fragile. While both phonetic symbolism (see Taylor \& Taylor, 1965) and automatic phonemic processing (e.g., Underwood \& Thwaites, 1982) have been demonstrated with visual presentations, the possibility remains that a stronger link to vowel frequency might emerge if the words employed here were presented auditorally.

\section{REFERENCES}

Brown, R. (1958). Words and things. Glencoe, IL: The Free Press. Craik, F. I. M., \& Tulving, E. (1975). Depth of processing and the retention of words in episodic memory. Journal of Experimental Psychology: General, 104, 268-294.

HeISE, D. (1965). Semantic differential profiles for 1,000 most frequent English words. Psychological Monographs, 79(8, Whole No. 601).

O'BOYLE, M., \& TARTE, R. (1980). Implications for phonetic symbolism: The relationship between pure tones and geometric figures. Journal of Psycholinguistic Research, 9, 535-544.

OsGOoD, C. (1962). Studies on the generality of affective meaning systems. American Psychologist, 17, 10-28.

PaIvio, A. (1975). Perceptual comparisons through the mind's eyes. Memory \& Cognition, 3, 635-647.

TARTE, R. (1982). The relationship between monosyllables and pure tones: An investigation of phonetic symbolism. Journal of Verbal Learning \& Verbal Behavior, 21, 352-360.

TARTE, R., \& BARritT, L. (1971). Phonetic symbolism in adult native speakers of English: Three studies. Language \& Speech, 14, 158-168.

TARTE, R., \& O'BoYLE, M. (1982). Semantic judgments of compressed monosyllables: Evidence for phonetic symbolism. Journal of Psycholinguistic Research, 11, 183-196.

TAYLOR, I. (1976). Introduction to psycholinguistics. New York: Holt, Rinehart, \& Winston.

TAYLOR, I., \& TAYLOR, M. M. (1965). Another look at phonetic symbolism. Psychological Bulletin, 64, 413-427.

UNDERWOOD, G., \& THWAITES, S. (1982). Automatic phonological coding of unattended printed words. Memory \& Cognition, 10, 434-442.

(Manuscript received for publication September 20, 1985.) 